\title{
Los efectos agudos de la contaminación del aire en la salud de la población: evidencias de estudios epidemiológicos
}

\author{
José Alberto Rosales-Castillo, Q FB, M en $C,{ }^{(1)}$ Víctor Manuel Torres-Meza, MC, M en $C{ }^{(1)}$ \\ Gustavo O laiz-Fernández, MC, MSP, (2) Víctor H. Borja-A burto, MC, MSP, Ph D.. ${ }^{(3)}$
}

\begin{abstract}
Rosales-Castillo JA, Torres-Meza VM, Olaiz-Fernández G, Borja-Aburto VH. Los efectos agudos de la contaminación del aire en la salud de la población: evidencias de estudios epidemiológicos. Salud Publica Mex 2001;43:544-555
\end{abstract} El texto completo en inglés de este artículo está disponible en: http://www.insp.mx/salud/index.html

\section{Resumen}

Objetivo. Sintetizar las evidencias de los efectos en la salud de la población por la exposición a contaminación del aire por ozono y partículas suspendidas. Material y métodos. A partir de las principales publicaciones internacionales y mexicanas, publicadas y referidas hasta junio del año 2000, se realizó un metanálisis para resumir los efectos reportados a través del empleo de modelos de efectos aleatorios. Resultados LoS resultados se expresaron como porcentajes de incremento por 10 unidades de concentración de $P M_{10}\left(\mu \mathrm{g} / \mathrm{m}^{3}\right)$ y ozono $(\mathrm{ppb})$. Entre los efectos de $\mathrm{PM}$ cabe destacar el efecto agudo en la mortalidad (0.96\%), hospitalizaciones (1.39\%), visitas a salas de urgencias (3.11\%), sintomas respiratorios (7.72\%), parámetros de función pulmonar (1.42\%), para capacidad vital forzada (CVF) y días de actividad restringida (7.74\%). Los efectos de la exposición a 0zono son igualmente significativos. Conclusiones. Estos resultados muestran el gran impacto que las concentraciones de contaminantes del aire podrían tener en la salud de las poblaciones urbanas de las grandes metrópolis. El texto completo en inglés de este artículo está disponible en: http:/ /www.insp.mx/salud/index.html

Palabras clave: meta-análisis; 0zono; $\mathrm{PM}_{10}$; efectos en la salud; contaminación del aire; México

\author{
Rosales-Castillo JA, Torres-Meza VM, \\ Olaiz-Fernández G, Borja-Aburto VH. \\ Acute effects of air \\ pollution on health: \\ Evidence from epidemiological studies. \\ Salud Publica Mex 2001;43:544-555. \\ The English version of this paper \\ is available at: http://www.insp.mx/salud/index.html
}

\begin{abstract}
Objective. To summarize the evidence of acute health effects from exposure to particulate matter and ozone. Ma terial and Methods A meta-analysis was performed using random effect models, to summarize the health effects of pollution, published in major international and Mexican journals up to June 2000. Results. Results were given as percent increase in ten units of PM10 $(\mu \mathrm{g} / \mathrm{m} 3)$ and ozone $(\mathrm{ppb})$. PM10 was found to have an effect on mortality $(0.96 \%)$, hospital admissions $(1.39 \%)$, emergency room visits $(3.11 \%)$, respiratory symptoms $(7.72 \%)$, pulmonary forced vital capacity (1.42\%), and restricted activity days (7.74\%). 0 zone effects were equally significant. Conclusions. These findings show the great impact that air pollutants may have on the health of po pulations living in large metro polis. The English version of this paper is available at: http://www.insp.mx/ salud/index.html
\end{abstract}

Key words: meta-analysis; ozone; $\mathrm{PM}_{10}$; health effects; air pollution; Mexico

Partes de este proyecto fueron subvencionadas por el Banco Mundial (World Bank) y por el IDRC (International Development Research Center) de Canadá.

(1) Dirección General de Salud Ambiental, Secretaría de Salud, México, D.F., México.

(2) Instituto $\mathrm{N}$ acional de Salud Pública, Cuernavaca, Morelos, México.

(3) Instituto Mexicano del Seguro Social, México, D.F., México.

Fecha de recibido: 24 de noviembre de 2000 - Fecha de aprobado: 11 de junio de 2001

Solicitud de sobretiros: Dr.Víctor H. Borja-Aburto. Centro Médico N acional Siglo XXI. Avenida Cuauhtémoc 330, colonia Doctores, 06725 México, D.F., México.

Correo electrónico: dpmcoost@msj.gob.mx 
L a contaminación atmosférica antropogénica ha venido acompañando al hombre prácticamente desde hace casi 500 años. El primer caso de efectos severos, reconocido históricamente fue el ocurrido en Londres en diciembre de 1952, donde los altos niveles de contaminantes se asociaron con un alarmante incremento en el número de muertes (alrededor de 4000). Otras ciudades donde se han presentado altos niveles de contaminantes del aire son: México, Río de Janeiro, Milán, Ankara, Melbourne, Búfalo, Tokio y Moscú, por nombrar algunas. ${ }^{1}$

Por fortuna, la calidad del aire ahora se vigila más y las altas concentraciones de contaminantes observadas en los años 60 y 70 ya no se presentan. No obstante, la exposición continua a moderados bajos niveles de contaminante por largos periodos de tiempo es un fenómeno que se da cotidianamente. Por esta razón ha cobrado una gran importancia determinar los efectos causados por estas exposiciones más bajas, así como los efectos por exposiciones prolongadas. Sin embargo, tratar de utilizar esta información no ha sido fácil, debido principalmente al gran número de informes de estudios epidemiológicos, no siempre concordantes, y las incertidumbres derivadas de la falta de identificación precisa de los agentes causales de estos efectos. ${ }^{2,3}$

Por ser contaminantes cuya vía de entrada al organismo es la inhalatoria, es de esperarse que sus principales efectos sean en las vías respiratorias. Así, los efectos más comúnmente reportados en la salud por exposición a la contaminación del aire son en vías respiratorias (bronquitis, enfermedad pulmonar obstructiva crónica, neumonía etc.), en el sistema cardiovascular (arritmias, infartos, etc.). De hecho, el efecto agudo en la mortalidad es el efecto tóxico que más se ha estudiado. ${ }^{4}$ Asimismo, en los últimos años se ha venido estudiando con más detalle a los grupos poblacionales que son más susceptibles: los niños, personas de la tercera edad y asmáticos.

El número de publicaciones de estudios sobre los efectos en la salud por contaminación del aire ha crecido durante la última década, por lo que el clásico resumen narrativo ya no es apropiado para dar los resultados en este campo. Por esta razón decidimos aplicar un metanálisis, cuya metodología se enfoca en combinar los resultados de los diferentes estudios para determinar patrones.

Por ser los dos contaminantes más importantes que se presentan en la ciudad de México y otras ciudades de América Latina, este análisis se enfocó en los estudios referentes a las partículas suspendidas (PS) y al ozono.

\section{Material y métodos}

A partir de una búsqueda exhaustiva en Medline, se identificaron los estudios que hubieran evaluado efectos en la salud por exposición a ozono y partículas suspendidas en poblaciones humanas, y que hubiesen sido publicados y referidos hasta junio del año 2000. Se incluyeron los artículos donde se hubieran estudiado la asociación entre la exposición a ozono o PS y algún efecto agudo en la salud de poblaciones tal como mortalidad, hospitalizaciones, visitas a salas de urgencias, efectos en individuos asmáticos, efectos en la población, parámetros de función pulmonar y días de actividad restringida. En su mayoría estos son estudios epidemiológicos que evalúan los efectos de la exposición en periodos cortos, de 1 a 5 días. Por ser otro tipo de estudios, con consecuencias diferentes, no se incluyeron los relacionados con efectos de la exposición crónica a contaminantes, como la disminución de la esperanza de vida o el desarrollo de enfermedad pulmonar obstructiva crónica o cáncer.

Los criterios para la inclusión y exclusión de los trabajos fueron los siguientes: los trabajos deberían ser de áreas geográficas definidas y con informes de mediciones de partículas, ya fueran suspendidas totales (PST), humo negro (BS), coeficiente de Haze $(\mathrm{CoH}) \mathrm{u}$ otra forma de cuantificar partículas suspendidas u ozono. Se excluyeron los artículos que no presentaran alguna medida de dispersión (intervalos de confianza, error estándar o varianza) para las asociaciones reportadas (porcentaje de cambio, riesgo relativo $-\mathrm{RR}-\mathrm{o}$ razón de momios -RM-) ni aquellos que en sus análisis no controlaran por el efecto de temperatura y variaciones estacionales en los intervalos de tiempo estudiados. No se incluyeron los efectos bioquímicos o moleculares, ni estudios en animales de laboratorio, por la dificultad de extrapolación a poblaciones humanas.

Para obtener estimaciones semejantes, se transformaron las diversas formas de cuantificar PS en su equivalente en $\mathrm{PM}_{10}$ (mezcla de diferentes componentes donde $50 \%$ del material sólido tiene un tamaño de $10 \mu \mathrm{m})$. Para esto se utilizaron las conversiones siguientes: $\mathrm{PM}_{10} \cong \mathrm{PM}_{15}, \mathrm{PM}_{10} \cong \mathrm{PM}_{13}, \mathrm{PM}_{10} \cong \mathrm{TSP}$ *0.55, $\mathrm{PM}_{10} \cong \mathrm{PM}_{2.5} / 0.6, \mathrm{PM}_{10} \cong \mathrm{BS}, \mathrm{PM}_{10} \cong \mathrm{CoH} / 0.55 .{ }^{5}$

De la misma manera, para dar uniformidad al reporte de resultados, los valores RR, RM, porcentajes de cambio, coeficientes de regresión (Poisson o logarítmica) se transformaron en porcentajes de cambio por 10 unidades de concentración de contaminante (para $\mathrm{PM}_{10} \mu \mathrm{g} / \mathrm{m}^{3}$ y para ozono $\mathrm{ppb}$ ). 
Para estimar un promedio ponderado de los efectos en la salud reportados a partir de los estudios seleccionados, con sus respectivos intervalos de confianza al $95 \%$, se emplearon modelos de efectos aleatorios, debido a que los modelos de efectos fijos consideran sólo errores de muestreo en los estudios, pero no consideran la heterogeneidad de muestreo de sitios diferentes. Este modelo asume que cada estudio tiene su propio efecto asociado a la exposición y que hay una distribución aleatoria alrededor de un valor central del efecto. ${ }^{6,7}$

Debido a que no existe un acuerdo en los efectos atribuidos a la exposición a ozono, y especialmente en el caso del efecto agudo sobre la mortalidad, se presenta un análisis por separado que muestra los resultados de aquellos estudios que controlaron el efecto de ambos contaminantes en modelos multivariados.

\section{Resultados}

Los cuadros I y II muestran la media obtenida de la estimación combinada de los estudios acerca de los efectos en salud seleccionados, con sus respectivos intervalos de confianza y las referencias de los mismos. A continuación hacemos una descripción de los resultados más relevantes.

\section{Mortalidad y exposición aguda a partículas suspendidas}

De los efectos tóxicos asociados a $\mathrm{PM}_{10}$, la mortalidad es, sin lugar a dudas, la más estudiada. Las publicaciones donde se ha notificado un incremento en la mortalidad asociada con los niveles de contaminación se han venido haciendo desde los años 50. Para efectos de este trabajo se incluyeron los principales estudios efectuados en los años 80 y 90, en América (34), Europa (15), Asia (2) y Oceanía (1). Todos estos estudios emplearon análisis de series de tiempo de la variación diaria concomitante entre la calidad del aire y el número de muertes diarias. La figura 1 muestra los estudios incluidos en el análisis de mortalidad. Como es de esperarse, las estimaciones para mortalidad por causas cardiovasculares $(1.32 \%)$ y mortalidad por causas respiratorias $(1.82 \%)$ son mayores que la estimada para mortalidad total $(0.96 \%)$ (cuadro I); estos resultados coinciden con las tendencias marcadas por Dockery y Pope en 1994. ${ }^{5}$ Entre ellos destacamos dos estudios realizados en América Latina. ${ }^{8,9}$

\section{Mortalidad infantil y exposición aguda a partículas suspendidas}

La mortalidad infantil asociada a la exposición a PS es un aspecto importante que, sin embargo, no ha sido ampliamente estudiado. La mayoría de los estudios de este campo son de corte transversal, como fueron los realizados por Bobak y colaboradores, en 1992,10 y el de Woodroff y colaboradores en 1997. ${ }^{11}$ Hasta 1999, sólo se reporta un trabajo de series de tiempo, realizado por Loomis y colaboradores en 1999 en la ciudad de México (México). ${ }^{12}$ En dicho estudio se encontró un incremento de $3.52 \%$ en la mortalidad de menores de un año.

\section{Mortalidad y exposición aguda a ozono}

Respecto a la mortalidad asociada a la exposición a ozono, en contraste con la exposición a las PS, los resultados han sido debatidos, principalmente porque en la mayoría de las ocasiones los contaminantes se presentan a altas concentraciones, simultáneamente. En un intento de diferenciar la importancia de las partículas suspendidas en las asociaciones entre el ozono y la mortalidad, se calcularon las estimaciones combinadas considerando todos los estudios $(0.99 \%)$ y una estimación restringida a los estudios que estimaran los efectos del ozono ajustando por PS en los modelos estadísticos $(0.59 \%)$, encontrándose una pequeña diferencia entre las dos aproximaciones (cuadro II).

\section{Hospitalizaciones y visitas a salas de urgencias por exposición aguda a partículas suspendidas}

El estudio de la mortalidad relacionada con la exposición a contaminantes del aire puede ser sólo el pico del iceberg de los efectos en la salud causados por los contaminantes. Es claro que por cada persona que fallece, hay muchas más que enferman. Por esta razón es importante considerar los resultados de estudios de hospitalizaciones y visitas a salas de urgencias. En los últimos años se han publicado diversos trabajos donde se reporta una asociación entre los niveles de PS y estos efectos en la salud por diversas causas, como son: respiratorias, asma, neumonía, cardiovasculares y cerebrovasculares.

Para este caso, cabe resaltar la estimación combinada para hospitalizaciones por asma (3.02\%), que fue 


\section{Cuadro I \\ Estimación combinada para diversos efectos en la SALUd asociados a La exposición a PM 10}

Efecto en la salud

1.1 Efecto agudo sobre la mortalidad

Total

Porcentaje de cambio ${ }^{1}$

0.96

$0.83-1.18$

\begin{tabular}{llll}
\hline Respiratoria & 1.82 & $1.37-2.22$ & $\begin{array}{l}8,9,13,30,31,34,40,41,43,43,43,45,47,48, \\
51,53,56,57,59,61,62\end{array}$ \\
& & & $8,9,13,30,31,33,34,40,42,43,43,43,45,45,46$, \\
& 1.32 & $1.10-1.55$ & $51,53,56,57,59$ \\
\hline Cardiovascular & & & $8,9,30,31,40,41,45,46,51,59,63$ \\
& 1.18 & $0.66-1.57$ & 12
\end{tabular}

1.2 Hospitalizaciones

\begin{tabular}{llll} 
Respiratorias & 1.39 & $1.18-1.60$ & $14,14,17,17,61,62,64,65,66,67,68,69$ \\
\hline Respiratorias $(>65)$ & 1.49 & $1.20-1.78$ & $14,14,66,70,70,71$ \\
\hline Asma & 3.02 & $2.05-4.00$ & $16,17,17,20,65,68,69,71,72,73,74$ \\
\hline N eumonía & 1.40 & $1.05-1.75$ & $48,71,75,76$. \\
\hline N eumonía $(>65)$ & 0.60 & $0.42-0.79$ & $15,65,69,77,78$. \\
\hline Cardio y cerebrovasculares & 1.22 & $0.94-1.50$ & 15,70
\end{tabular}

1.3 Visitas a salas de urgencias

\begin{tabular}{llll} 
Causas respiratorias & 3.11 & $2.35-3.88$ & $18,79,80,81$ \\
\hline Asma & 4.50 & $2.16-7.00$ & 16
\end{tabular}

1.4 Efectos en asmáticos

$\begin{array}{llll}\text { A taques de asma } & 7.87 & 4.48-11.27 & 64,74,82,83,84,85\end{array}$

$\begin{array}{llll}\text { Ataques de asma y uso de broncodilatador } & 10.22 & 7.30-13.14 & 19,83,86,87,88 \text {. }\end{array}$

\begin{tabular}{llll}
\hline Tos sin flemas (niños) & 4.54 & $2.65-6.44$ & $20,87,88,89,90$. \\
\hline
\end{tabular}

$\begin{array}{llll}\text { Tos con flemas (niños) } & 3.32 & 2.01-4.64 & 20,87\end{array}$

1.5 Síntomas en vías respiratorias en la población general

\begin{tabular}{lrrr} 
Vías respiratorias & 7.72 & $0.61-14.84$ & \multicolumn{1}{l}{$91,92,93$} \\
\hline Vías respiratorias altas & 4.39 & $3.56-5.12$ & $22,90,94$ \\
\hline Vías respiratorias bajas & 6.85 & $5.16-8.54$ & $20,22,86,90,94$ \\
\hline Bronquitis & 11.00 & $8.96-13.58$ & $64,91,93,95,96$
\end{tabular}

1.6 Parámetros de función pulmonar

\begin{tabular}{llll} 
VEF & -1.58 & -2.13 a -0.85 & $25,97,98,99,100,101$ \\
\hline CVF & -1.43 & -1.64 a -1.22 & 25,97 \\
\hline FMME & -8.00 & $-5.00 \mathrm{a}-11.00$ & 102 \\
\hline FEM & -0.39 & $-0.48 \mathrm{a}-0.31$ & $19,20,23,83,90,92,99,102,103$
\end{tabular}

1.7 Días de actividad restringida y días de actividad restringida menor

\begin{tabular}{lll} 
DAR & 7.74 & 104 \\
\hline DARM & 4.92 & 26
\end{tabular}

1 Porcentaje de cambio por $10 \mu \mathrm{g} / \mathrm{m}^{3}$

VEF: volumen espiratorio forzado en un segundo, CVF = Capacidad vital forzada, FMME: flujo máximo a media espiración, FEM: flujo espiratorio máximo DAR: días de actividad restringida, DARM: días de actividad restringida menor 


\section{Cuadro II \\ Estimación combinada para diversos efectos en la salud asociados a la exposición a Ozono}

Efecto en la salud

1.1 Efecto agudo sobre la mortalidad Total (no ajustada por partículas) Total (ajustada por partículas) Respiratoria

C ardiovascular

Personas $>65$

1.2 Hospitalizaciones

\begin{tabular}{llll} 
Total & 1.74 & $1.16-2.32$ & 17,68 \\
\hline Respiratorias & 3.76 & $0.45-7.05$ & $14,66,69$ \\
\hline Respiratorias $(>65)$ & 2.83 & $1.71-3.95$ & $66,67,71,110$. \\
\hline Asma & 1.47 & $0.41-2.53$ & $17,68,69$ \\
\hline N eumonía & 5.20 & $2.60-8.00$ & 111 \\
\hline N eumonía $(>65)$ & 0.98 & $0.53-1.43$ & 69
\end{tabular}

1.3 Visitas a salas de urgencias

\begin{tabular}{llll} 
Causas respiratorias & 3.17 & $1.67-4.67$ & $18,110,112,113$ \\
\hline Asma & 3.50 & $1.70-5.30$ & 114 \\
\hline Traqueítis & 12.5 & $0.0-29.16$ & 80
\end{tabular}

1.4 Efectos en asmáticos

\begin{tabular}{llll} 
Ataques de asma & 2.45 & $0.00-5.90$ & 96 \\
\hline Ataques de asma y uso de broncodilatador & 1.80 & $0.20-3.60$ & 21 \\
\hline Vías respiratorias bajas & 0.23 & $0.14-0.33$ & 115 \\
\hline Síntomas respiratorios (niños) & 0.66 & $0.55-0.76$ & $20,21,86,115$
\end{tabular}

1.5 Síntomas en vías respiratorias en la población general

$\begin{array}{llll}\text { Vías respiratorias altas } & 1.50 & 1.10-2.20 & 116 \\ \text { Vías respiratorias bajas } & 2.20 & 1.10-3.40 & 22\end{array}$

1.6 Parámetros de función pulmonar

\begin{tabular}{llll} 
FEM & -1.15 & -2.32 a -0.02 & $20,24,103$ \\
\hline FEV & -4.97 & -9.89 a -0.06 & 24,25 \\
\hline CVF & -4.77 & -9.47 a -0.07 & 24,25
\end{tabular}

1.7 Días de actividad restringida menor DARM

26

1 Porcentaje de cambio por $10 \mathrm{ppb}$

VEF: volumen espiratorio forzado en un segundo, CVF: capacidad vital forzada, FEM: flujo espiratorio máximo, D AR: días de actividad restringida, D ARM: días de actividad restringida menor

la más alta: probablemente esto se pueda fundamentar en el mecanismo de esta enfermedad (cuadro I).

En particular, Schouten y colaboradores en 1996, ${ }^{14}$ como parte del proyecto Air pollution on emergency hospital admissions (APEHA), realizaron un estudio en las dos ciudades holandesas más importantes: Amsterdam y Rotterdam, donde en individuos de entre 15 y 64 años hubo un incremento de $3.83 \%$ en las hospitalizaciones por causas respiratorias. Schwartz en $1997^{15}$ realizó un estudio de series de tiempo en Tucson-Arizona, Estados Unidos de América (EUA), observando la relación entre las hospitalizaciones por causas cardiovasculares en individuos mayores de 65 años con los niveles de contaminantes, encontrando un incremento de $1.19 \%$ en las admisiones hospitalarias por dichas causas. 


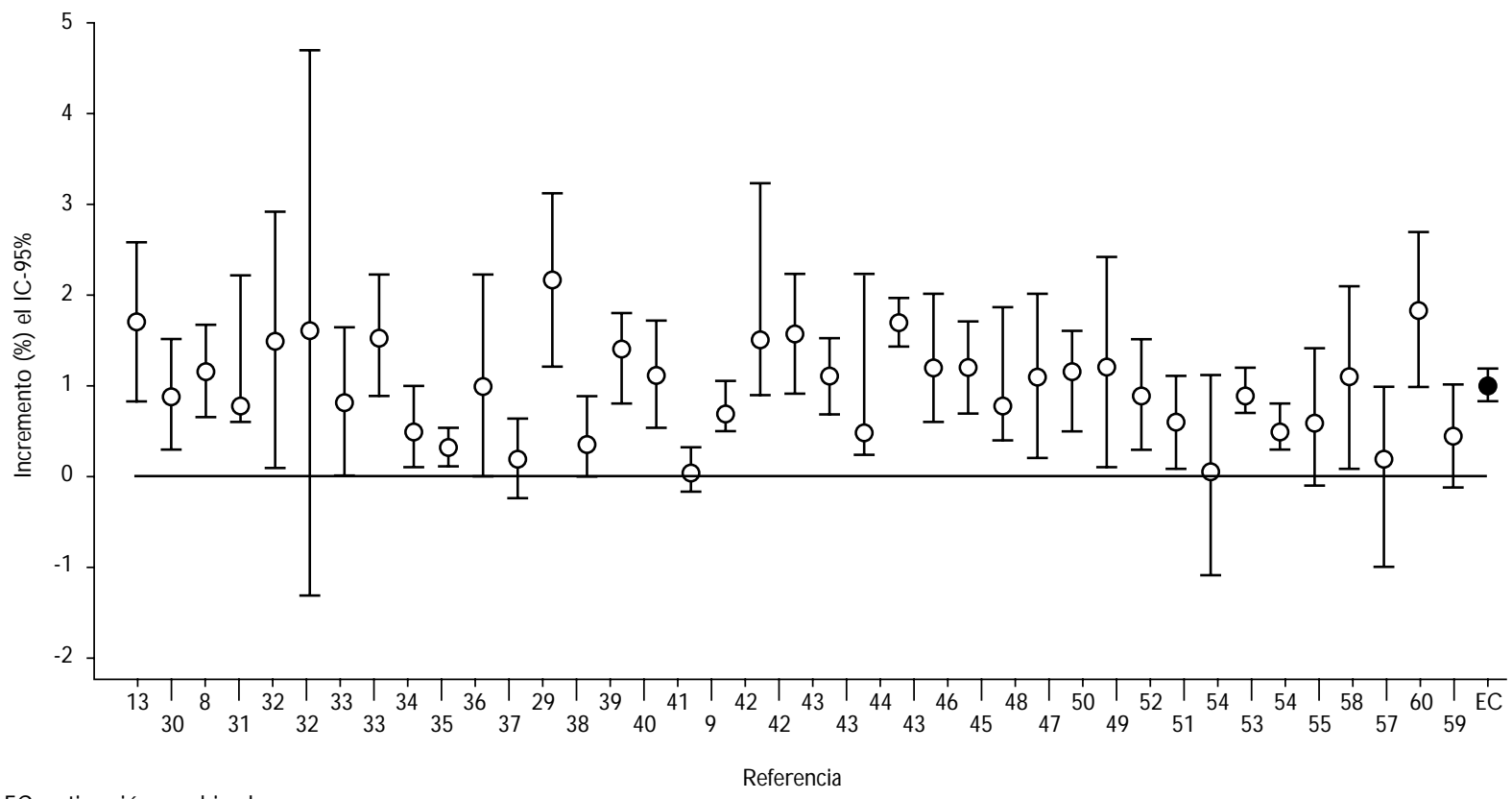

EC: estimación combinada

Figura1. Cambio porcentual en la mortalidad general (excluyendo muertes Violentas) por Cada $10 \mu \mathrm{\mu g} / \mathrm{M}^{3}$ de $\mathrm{PM}_{10}$

En el año de 1997 Lippset y colaboradores ${ }^{16}$ realizaron un conteo de las visitas a salas de urgencia (VSU) por ataques de asma en Santa Clara-California durante el invierno. Los resultados mostraron un incremento significativo de $4.5 \%$, ajustando el modelo con una temperatura de $30^{\circ} \mathrm{F}$. Cabe hacer mención que los individuos que sufren asma, así como los niños y personas de la tercera edad, son especialmente susceptibles a sufrir los efectos tóxicos de la contaminación.

\section{Hospitalizaciones y visitas a salas de urgencias por exposición aguda a Ozono}

En contraste con lo encontrado para PS, las estimaciones combinadas con los mayores incrementos fueron las asociadas a causas respiratorias (cuadro II). Entre los estudios incluidos tenemos el que realizaron Thurston y colaboradores en $1992^{17}$ en diversas ciudades del estado de Nueva York (Buffalo, Albany, Whitte Plain y Nueva York) donde observaron un incremento de 5\% en las hospitalizaciones por asma. En Canadá se han hecho diversos estudios buscando incrementos en las VSU por exposición a contaminantes, de éstos destacamos el de Delfino y colaboradores, ${ }^{18}$ en Montreal, y para el cual utilizaron un modelo de series de tiempo y encontraron un incremento de 7\% en las VSU por causas respiratorias.

\section{Síntomas respiratorios y exposición aguda a partículas suspendidas}

El uso de registros diarios de síntomas respiratorios es una forma menos costosa de evaluar el efecto en la salud, principalmente en vías respiratorias, por exposición aguda a PS. Los síntomas más comunes son: los de vías respiratorias altas (tos con flemas, fiebre, sinusitis, etc.) y bajas (tos seca, respiración con dolor, resfriado, etc.). Cabe mencionar que la tos es el síntoma más común ${ }^{5}$ y que la población más comúnmente estudiada en este tipo de trabajos es la escolar. Para efectos de esta investigación, los síntomas en vías respiratorias se clasificaron en dos sectores poblacionales: asmática y general. Las estimaciones combinadas con los mayores incrementos fueron los ataques de asma y uso de broncodilatador $(10.22 \%)$ para la población asmática, y bronquitis para la población general (11\%) (cuadro I).

En particular, mencionaremos los estudios realizados por Pope y colaboradores ${ }^{19}$ en 1991, y el de Romieu y colaboradores en $1996 .{ }^{20}$ En el primero, usan- 
do un modelo de series de tiempo, se informa un incremento de $5 \%$ en los síntomas en vías respiratorias altas; cabe hacer mención que para este estudio se empleó una población escolar y el modelo se ajustó por temperatura. En el segundo, en una población de niños con diagnóstico de asma, entre 5 y 13 años, del Hospital Infantil de México (Ciudad de México) se encontró un incremento en la presencia de síntomas en vías respiratorias bajas de $12.6 \%$.

\section{Síntomas respiratorios y exposición aguda a ozono}

Los efectos en la salud asociados con la exposición a ozono son semejantes a los encontrados para PS, pero con estimaciones combinadas resultan más pequeños. La estimación combinada para ataques de asma (2.45\%) resultó ser la mayor en esta clasificación (cuadro II). En este rubro resaltamos el estudio de Hiltermann de $1998^{21}$ donde, en una población de individuos asmáticos seleccionada de la Clínica de la Universidad de Leiden, encontraron un incremento en la presencia de respiración corta de $3.4 \%$, usando un modelo sin rezago (lag 0). Ostro en $1993^{22}$ estudió un grupo de no fumadores en el sur de California (EUA) y encontró un incremento de $2.2 \%$ en presencia de síntomas en vías respiratorias bajas.

\section{Parámetros de función pulmonar y exposición a partículas suspendidas}

No siempre la sintomatología o la ocurrencia de ciertas enfermedades son utilizadas como parámetros para determinar la toxicidad de los contaminantes atmosféricos. En muchas ocasiones es necesario determinar algún parámetro que nos permita establecer precozmente los efectos tóxicos sin esperar a que aparezca clínicamente la enfermedad, y por ello se ha tratado de establecer la asociación entre los niveles de contaminación y algunos parámetros espirométricos como volumen espiratorio forzado (VEF), capacidad vital forzada (CVF), flujo máximo a media espiración (FMME) y flujo espiratorio máximo (FEM). Los decrementos porcentuales de las estimaciones combinadas para estos parámetros van desde $-0.39 \%$ (VEF) hasta un $-1.58 \%$ (FEV) (cuadro I)

Entre los estudios incluidos tenemos el de Neas y colaboradores de $1996,{ }^{23}$ en el cual se usó una cohorte de 108 niños de Pennsylvania (EUA); este estudio lo llevó a cabo durante el verano de 1991, encontrando una disminución en el FEM de $0.15 \%$. En el mismo sentido Romieu y colaboradores ${ }^{20}$ en el mismo año, en una población de niños asmáticos, encontraron una disminución para este mismo parámetro espirométrico de $0.82 \%$.

\section{Parámetros de función pulmonar y exposición a ozono}

Nuestras estimaciones combinadas para estos efectos resultaron mayores para ozono que las de PS, entre $-1.15 \%$ (FEM) y $-4.97 \%$ (VEF) (cuadro II).

De manera particular, en el estudio de Castillejos y colaboradores de $1992,{ }^{24}$ realizado en la ciudad de México (México), concretamente en la zona suroeste (El Pedregal), en niños entre 7 y 9 años, se determinó una disminución de 1.22\% en el FEM. En el mismo sentido, Brunekreef y colaboradores en $1991^{25}$ llevaron a cabo un estudio en diferentes comunidades del estado de Ohio (EUA), donde seleccionaron una población escolar; en este caso se encontró una reducción de 1.29\% en CVF.

\section{Días de actividad restringida y exposición a partículas suspendidas y ozono}

Desde un punto de vista económico los días en los cuales un trabajador detiene sus actividades o baja su productividad (DAR y DARM, respectivamente), debido a que padece alguna enfermedad asociada a la exposición a contaminantes es un importante factor, pues esto se traduce en una pérdida en la productividad económica. En la actualidad existen pocos estudios sobre este parámetro; destacamos el de Ostro en $1989,{ }^{26}$ el cual hizo en varias ciudades de EUA durante 1976 a 1981 e incluyendo a las PS y al ozono como contaminantes. En este trabajo se determinaron incrementos de $7.74 \%$ y $4.92 \%$, en el DAR y DARM respectivamente, para PS; y de $18.5 \%$ para DARM, asociado con la exposición a ozono.

\section{A usentismo escolar y exposición a partículas suspendidas}

Otro parámetro asociado con una disminución en la actividad de las personas, concretamente con la de los niños, y que se ha visto está asociado con los altos niveles de contaminación por $\mathrm{PM}_{10}$, es el ausentismo escolar, el cual indirectamente nos marca el efecto en la salud causado por esta exposición. Sobre esto se tienen muy pocos reportes; destacan el estudio realizado por Ransom y colaboradores en $1992^{27}$ en UTAH (EUA) y en éste se reporta un incremento de $0.21 \%$ en los días que faltan los niños por enfermedades relacionadas con los altos niveles de partículas, y el de Romieu y colaboradores en $1992^{28}$ en la Ciudad de México (México); 
durante tres meses se siguió a los infantes que acudían a un jardín de niños de la zona suroeste de la ciudad, determinándose un incremento en la exposición dependiente con los niveles de ozono; entre los resultados destaca un incremento de $3.4 \%$ en el ausentismo por cada 10 ppb de ozono, por enfermedades respiratorias asociadas a la exposición a este contaminante.

\section{Discusión}

Los resultados anteriores muestran una gran coherencia entre los efectos estudiados, desde efectos subclínicos, síntomas, uso de servicios de salud por causas respiratorias y cardiovasculares, hasta el incremento en el número de muertes. La coherencia de la asociación entre los efectos hace plausible las asociaciones encontradas con estos contaminantes del aire, lo cual se ve respaldado con los resultados de algunos estudios clínicos y toxicológicos donde han encontrado, entre otras cosas: una disminución de la función pulmonar, incremento en la frecuencia de síntomas respiratorios, hipersensibilidad exaltada celular y en vías respiratorias, y evidencia bioquímica de inflamación en pulmón, que en parte podrían explicar el mecanismo de daño producido por los contaminantes. ${ }^{117} \mathrm{Sin}$ embargo, todavía existe un debate sobre la causalidad de estas asociaciones y cuál es el contaminante responsable de estos efectos.

La investigación sobre los mecanismos por los cuales los diferentes contaminantes producen sus efectos tóxicos está todavía en etapas tempranas, pero lo que sí es claro es que no todos los contaminantes atmosféricos tienen la misma capacidad de producir efectos tóxicos ni producen el mismo daño, por lo que es lógico pensar que estas diferencias se deben a las propiedades físicas y químicas de dichos componentes. En este sentido, en algunos estudios se ha observado que las sustancias que se encuentran en forma de aerosol son más tóxicas que los compuestos que se encuentran en forma de gas. Esto probablemente se debe a que los compuestos en estado gaseoso son eliminados de las vías respiratorias mucho más fácilmente que los aerosoles, los cuales son absorbidos o depositados más rápidamente. ${ }^{117}$

En relación con las PS se ha observado que conforme disminuye el tamaño de partícula aumenta su toxicidad, esto se debe a que las partículas más pequeñas son capaces de penetrar más profundamente en las vías respiratorias y una vez dentro del árbol respiratorio éstas son capaces de acumularse en diferentes sitios, como fosas nasales, laringe, tráquea, bronquios, bronquiolos y sacos alveolares. ${ }^{118,119}$ Aunque también son importantes los componentes que con- forman de las PS, se ha observado que el aire en donde son más abundantes los álcalis suele producir efectos tóxicos más severos que cuando se encuentran principalmente componentes ácidos; ${ }^{3,117,120}$ en el mismo sentido, se ha visto que el potencial redox se ha asociado con el desarrollo de alveolitis neutrofílica, reacciones de hipersensibilidad e incrementos en las infecciones pulmonares. ${ }^{117,119}$

Sin embargo, nuestro conocimiento sobre los mecanismos biológicos es aún limitado. En este sentido, un hecho que resulta interesante de analizar es que la plausibilidad biológica de los efectos se incrementa y se sustenta en una cascada de eventos cardiopulmonares y porque la mayoría de los efectos cardiopulmonares están asociados con la exposición a PS. Por ejemplo, un mecanismo de daño a pulmón (incluyendo un potencial daño oxidativo y por inflamación), donde se disminuye la función pulmonar, y la enfermedad cardiovascular, están potencialmente relacionados con la hipoxemia. Aunque en un estudio donde se asociaron hipoxemia y los niveles de contaminante no se encontró una disminución en la saturación de oxígeno en sangre. ${ }^{121} \mathrm{El}$ sistema nervioso autónomo puede jugar un importante papel en el mecanismo fisiopatológico; se ha hipotetizado que las PS pueden provocar un proceso inflamatorio a nivel alveolar, lo que origina secreción de citocinas potencialmente dañinas, así como incremento en la coagulabilidad de la sangre. ${ }^{122}$ Cambios activados por el sistema nervioso autónomo, que incrementan la viscosidad de la sangre pueden afectar la variabilidad y frecuencia del ritmo cardiaco, ${ }^{123-125}$ lo que puede aumentar la posibilidad de morir. ${ }^{126}$ Sin embargo la mayoría de estos trabajos han sido de tamaño muy limitado.

El ozono es un gas altamente oxidante y es parcialmente eliminado en las vías respiratorias altas cuando es exhalado, pero una importante fracción de éste alcanza las vías respiratorias bajas. Hay algunos investigadores que han propuesto que algunos de los efectos tóxicos del ozono son causados por su capacidad oxidativa, ${ }_{1}^{127}$ pero algunos científicos han calculado que sólo una pequeña parte de este gas alcanza la membrana apical del epitelio celular en vías respiratorias y que sus efectos tóxicos se deben a sus productos de reacción: los hidroxihidroperóxidos y los hidroxialdehidos. ${ }^{127}$

Por otro lado, el uso de los metanálisis como metodología para encontrar patrones consistentes, es una metodología útil que, sin embargo, tiene limitaciones que deben tomarse en cuenta en la interpretación de los resultados finales. La transformación de las unidades de medida de los contaminantes a una sola 
medida común parte de supuestos de equivalencias que no necesariamente son iguales en todas las localidades donde se originaron los estudios, y, por otro lado, la transformación de una medida común a incrementos porcentuales, a partir de distintos diseños epidemiológicos y distintos modelos estadísticos empleados, tiene también sus limitaciones; además, cuando existe heterogeneidad entre los estudios el empleo de modelos de efectos aleatorios sólo toma en cuenta la variación estadística de los resultados de los estudios provenientes de diferentes ciudades y no las diferencias que pueden ser atribuibles a efectos diferentes por diferencial de los contaminantes, ya sea por su composición o por características sociodemográficas distintas de las poblaciones. Sin embargo, a pesar de estas incertidumbres, en el análisis de riesgos de los contaminantes es necesario tener resultados cuantitativos a partir de varios estudios, principalmente cuando éstos son realizados en ciudades donde la información requerida es limitada, como sería el caso particular de la Ciudad de México, por lo que los resultados obtenidos a partir de este metanálisis pueden tomarse como guía para la estimación de los efectos asociados a la exposición, y como referencia en la toma de decisiones, mientras no se cuente con información local completa.

\section{A gradecimientos}

Agradecemos los valiosos comentarios del doctor Germán Corey a este trabajo.

\section{Referencias}

1. D ockery DW, Pope CA III. Epidemiology of acute health effects: Summary of time-series studies. En:W ilson R, Spengler JD, ed. Particles in our air: Concentrations and health effects. $\mathrm{C}$ ambridge (MA): Harvard University Press, 1996:123-147.

2. Möller $L$, Schuetzle $D, A$ utrup H. Future research needs associated with the assessment of potencial human health risk from exposure to toxic ambient air pollutants. Environ Health Perspect 1994; 102 Suppl 4:193210.

3. Schlesinger R. Toxicological evidence for health effects from inhaled particulate pollution: Does it support the human experience? Inhal Toxicol 1995;7:99-109.

4. Schwartz J.Air pollution and daily mortality:A review and meta-analysis. Environ Res 1994;64:36-52.

5. Dockery D, Pope III C.Acute respiratory effects of particulate air pollution. Annu Rev Public Health 1994;15:107-132.

6. Blettner M, Sauerbrei W, Schlehofer B, Scheuchenpflug T, Friedenreich C. Traditional reviews, meta-analysis and pooled analysis in epidemiology. Int J Epidemiol 1999;28:1-9.

7. DerSimonian R, Laird N. Meta-analysis in clinical trials. Control Clin Trials 1986;7:177-188.
8. Borja-A burto V, Loomis D,BangdiwalaS,Shy C, Rascón-Pacheco R. 0 zone, suspended particulates, and daily mortality in Mexico City.Am J Epidemiol 1997; 145:258-268

9. 0 stro B, Sánchez J, Aranda C, Eskeland G. Air pollution and mortality: Results from a study of Santiago, Chile. J Expo Anal Env Epid 1996;6: 97-114.

10. Bobak M, León D.Air pollution and infant mortality in the Czech Republic, 1986-88. Lancet 1992;340:1010-1014.

11. W oodruff T, G rillo J, Schoendorf K.The relationship between selected causes of post neonatal infant mortality and particulate air pollution in the United States. Environ Health Perspect 1997;105:608-612.

12. Loomis D, C astillejos M, Gold D, MCD onnell W, Borja-A burto V. Air pollution and infant mortality in Mexico City. Epidemiology 1999;10: 118123.

13. Anderson H, Ponce de León A, Bland J, Bower J, Strachan J. Air pollution and daily mortality in London: 1987-92. Brit Med J 1996;312:665-669. 14. Schouten J,Vonk J, GraffA. Short term effects of air pollution on emergency hospital admissions for respiratory diseasse: Results of the APEHA project in two major cities in the Netherlands, 1977-89. J Epidemiol Commun H 1996; 50 Suppl1: S22-S30.

15. Schwartz J. Air pollution and hospital admissions for cardiovascular disease in Tucson. Epidemiology 1997; 8:371-377.

16. Lipsset $M$, Hurley S, 0 stro B.A ir pollution and emergency room visits for asthma in Santa Clara County California. Environ Health Perspect 1997;105:216- 222.

17.Thurston G, Kazahiko I, Patrick L, Kinney L. A multi-year study of air pollution and respiratory hospital admissions in three N ew York state metropolitan areas: Results for 1988 and 1989 summers. J Expo Anal Env Epid 1992;2:429-450.

18. Delfino R, Murphy-Moulton A, Burnett R, Brook J, Becklake M. Effects of air pollution on emergency room visits for respiratory illnesses in Montreal, Q uebec. Am J Respir C rit C are 1997;155:568-576.

19. Pope III A, D ockery D, Spengler J, Raizenne M. Respiratory health and $\mathrm{PM}_{10}$ pollution. A daily time series analysis. Am Rev Respir D is 1991; 144 668-674.

20. Romieu I, Meneses F, Ruiz S, Sienra J, Huerta J,W hite M. Effects of air pollution on the respiratory health of asthmatic children living in Mexico City.Am J Respir C rit C are 1996;154:300-307.

21. Hiltermann T, Stlok J, Z ee S, Brunekreef B, Bruijne C, Fischer P et al. Asthma severity and susceptibility to air pollution. Eur Respir J 1998;11: 686-693.

22. 0 stro B, Lipsett M, Mann J, Krupnick A, Harrington W. Air pollution and respiratory morbidity among adults in Southern California. Am J Epidemiol 1993;137:691-700.

23. N eas L, D ockery D, Burge H, Koutrakis P, Speizer F. Fungus spores, air pollution, and other determinants of peak espiratory flow rate in children. Am J Epidemiol 1996;143:797-807.

24. Castillejos M, Gold D, D ockery D, Tosteson D, Baum, D, Speizer E. Effects of ambient ozone on respiratory function and symptoms in Mexico C ity schoolchildren. Am Rev Respir D is 1992;145:276-282.

25. Brunekreef B, Kinney P,W are P, D ockery D, Speizer F, Spengler J et al. Sensitive subgroups and normal variation in pulmonary function response to air pollution episodes. Environ Health Perspect 1991;90:189-193.

26. 0 stro B. Air pollution and acute respiratory morbidity: An observational study of multiple pollutants. Environ Res 1989; 50: 238-247.

27. Ransom M, Pope III C. Elementary school absences and PM ${ }_{10}$ pollution in Utah Valley. Environ Res 1992;58:204-219.

28. Romieu I, Cortés-Lugo M, Ruiz-Velasco S, Sánchez S, Meneses F, Hernández M. Air pollution and school absenteeism among children in Mexico City.Am J Epidemiol 1992;136:1524-1531.

29. Mazumdar S, Sussman N . Relationships of air pollution to health: Results from the Pittsburgh Study.Arch Environ Health 1983;38:17-24. 
30. Ballester F, Corella D, Pérez-H oyos S, Hervás A.Air pollution and mortality in Valencia, Spain:A study using the APEHA methodology.J Epidemiol Commun H 1996;50:527-533.

31. Bremner S,Anderson H,Atkinson R, M cMichael A, Strachan D, Blandi J et al. Bower J.Short term associations between outdoor air pollution and mortality in London 1992-4. 0 ccup Environ Med 1999;56:237-244.

32. Dockery D, Schwartz J, Spengler J. Air pollution and daily mortality: Associations with particulates and acid aerosols. Environ Res 1992;59: 362-373.

33. Gamble J, Lewis J. Health and respirable paticulate (PM 10) air pollution: A causal or statistical associations? Environ Health Perspect 1996; 104:838-850.

34. Ito $K$, Thurston G. Daily $\mathrm{PM}_{10} /$ mortality associations: An investigation of at risk subpopulations.J Exp A nal Env Epid 1996;6:79-95

35. Kelsall J, Samet J,Zeger S, X u J.Air pollution and mortality in Philadelphia, 1974-1988. Am J Epidemiol 1997;146:750-62.

36. Kinney P, Ito K, Thurston G. A sensitivity analysis of mortality/PM-10 associations in Los Angeles. Inhal Toxicol 1995;7:59-69.

37. Lee J, Schwartz J. Reanalysis of the effects of air pollution on daily mortality in Seoul, Korea: A case-crossover design. Environ Health Perspect 1999;107:633-636.

38. Moolgavkar S, Luebeck E.A critical review of the evidence on particulate air pollution and mortality. Epidemiology 1996;7:420 -428.

39. Moolgavkar S, Luebeck E, Hall T, Anderson E. Air pollution and daily mortality in Philadelphia. Epidemiology 1996;6:476-484.

40. N eas L, Schwartz J, Dockery D.A case-crossover analysis of air pollution and mortality in Philadelphia. Environ Health Perspect 1999;107:629631.

41. 0 stro B. Fine particulate air pollution and mortality in two Southern California Counties. Environ Res 1995;70:98-104.

42. Pope III A, Kalkstein L. Synoptic weather modelling and estimates of the exposure-response relationship between daily mortality and particulate air pollution. Environ Health Perspect 1996;104:414-420.

43. Pope III A, Hill R,Villegas G. Particulate air pollution and daily mortality on Utha's W asatch front. Environ Health Perspect 1999;107:567-573.

44. Samet J, Zeger S, Kelsall J, X U J, Kalkstein L. Does weather confo und or modify the association of particulate air pollution with mortality? Environ Res 1998;77: 9-19.

45. Schwartz J.Total suspended particulate matter and daily mortality in Cincinnati, 0 hio. Environ Health Perspect 1994;102:186-189.

46. Schwartz J, D ockery D. Increased mortality in Philadelphia associated with daily air pollution concentrations. Am Rev Respir $D$ is 1992. 145:600604.

47. Schwartz J, D ockery D. Particulate air pollution and daily mortality in Steubenville, 0 hio. Am J Epidemiol 1992;135:12-19.

48. Schwartz J. Air pollution. and daily mortality in Birmingham Alabama. Am J Epidemiol 1993;137:1136-1147.

49. Schwartz J. Particulate air pollution and daily mortality in D etroit. Environ Res 1991;56:204-213.

50 . Schwartz J, Dockery D,N eas L. Is daily mortality associated specifically with fine particles? J Air W aste Mannage 1996;46:927-939.

51. Simpson R.W illiams G. Petroeschevsky A. Morgan G. Rutherford S. Associations between outdoor air pollution and daily mortality in Brisbane, Australia. Arch Environ Health 1997;52(6):442-454.

52. Spix C, Heinrich J, D ockery D, Schwartz J,Völksch G, Schwinkowski K et al.Air pollution and daily mortality in Erfurt, East Germany, 1980-1989. Environ Health Perspect 1993;101:518-526.

53. Sunyer J, Castellsagué J, Sáez M, Tobías A, Antó J. Air pollution and mortality in Barcelona.J Epidemiol Community Health 1996; 50:576-580. 54. Touloumi G, Pocock S, Katsouyanni K, Trchopolous D. Short-term effects of air pollution on daily mortality in Athens: A time series analysis. Int J Epidemiol 1994;23:957-967.
55.Touloumi G, Samoli E, Katsouyanni K. Daily mortality and "winter type" air pollution in Athens, Greece. A time series analysis within the APEHA Project.J Epidemiol Community Health 1996;50 Suppl1: S47-S51.

56. Verhoeff A, Hoek G, Schwartz J,Van W ijnen J. Air pollution and daily mortality in A msterdam. Epidemiology 1996;7:225-230.

57. W ordley J,W alters J, Ayres J. Short term variations in hospital admissions and mortality and particulate air pollution. 0 ccup Environ Med 1997:54:108-116.

58. Z mirou D, Barumandzadeh T, Balducci F, Ritter P, Laham G, C hilardi J Short term effects of air pollution on mortality in the city on Lyon, France, 1985-90. J Epidemiol Community Health 1996;50 Suppl 1:S30-S35.

59. C astillejos M, Borja-A burto V, D ockery D, Gold D, Loomis D.Airborne coarse particles and mortality. Inhal Toxicol 2000;12 Suppl 1:61-72.

60. Cropper L, Simon N, Alberinni A, Sharma P. The health effect of air pollution in Delhi, India. In Press 2000.

61. Dab W, Medina S, Q uenel P, Le Moullec Y, Le Tertre A, Thelot B et al. Short term respiratory health effects of ambient air pollution: Results of the APHEA project in París.J Epidemiol Community Health 1996;50 Suppl 1:S42-S46

62. Vigotti M, Rossi G, Bisanti L, Zanobetti A, Schwartz J. Short term effects of urban air pollution on respiratory health in Milan, Italy, 1980-89.J Epidemiol Community Health 1996;50 Suppl 1:S71-S75.

63. Saldiva P, Lichtenfels A, Palva P, Martins M, Massad E, Pereira S et al. Association between air pollution and mortality due to respiratory diseases in children in São Paulo, Brazil: A preliminary report. Environ Res 1994; 65:218-25

64. A bbey $D, W$ ang $B$, Burchette R, Vancuren T, Mills P. Estimated longterm ambient concentrations of $\mathrm{PM}_{10}$ and development of respiratory symptoms in a non smoking populations.Arch Environ Health 1995;50(2): 139-145.

65. Burnett R, Robert T, Daniel D,Krewski R, Dann T, Brook J.Associations between ambient particulate sulphate and admissions to 0 ntario hospitals for cardiac and respiratory diseases. Am J Epidemiol 1995;142(1): 15- 22.

66. Ponce de León A, Anderson J, Bland M, D avid P, Strachan P, Bower J. Effects of air pollution on daily hospital admissions for respiratory disease in London between 1987-88 and 1991-92.J Epidemiol Community Health 1995; 33 Suppl 1:S63-S70.

67. Schwartz J, Spix C, Touloumi G, Bachárová L, Barumamdzadeh T, Tertre A. Methodological issues in studies or air pollution and daily counts of deaths or hospital admissions. J Epidemiol Community Health 1996;50(1):S3-S11.

68. Thurston G, Kazahiko I, Hayes C , Bates D, Kinney P, Lippmann M. Respiratory hospital admissions and summertime haze air pollution in Toronto, 0 ntario: Consideration of the role of acid aerosols. Environ Res 1994:65:271-290.

69. Linn W, Szlachic Y, Gong H, Kinney P, Berhane K. Air pollution and daily hospital admissions in metropolitan Los Angeles. Environ Health Perspect 2000;108 (5):427-434.

70. Schwartz J. Air pollution and hospital admissions for heart disease in eight U.S. Counties. Epidemiology 1999;10:17-22.

71. Schwartz, J. Short term fluctuations in air pollution and hospital admissions of the elderly for respiratory disease.Thorax 1995;50:531-538.

72. Delfino R, Becklarke M, Hanley J.The relationships of urgent admissions for respiratory illness to photochemical air pollution levels in Montreal. Environ Res 1994; 67:1-19.

73. 0 stro B, Lipsett $M, W$ iener $M$, Selner J. Asthmatic responses to airborne acid aerosols. Am J Public Health 1991;81:694-702.

74. Sheppard L, Levy D, N orris G, Larson T, Koenig Q . Effects of ambient air pollution on non elderly asthma hospital admissions in Seattle, W ashington, 1987-1994. Epidemiology 1999;10:23-30. 
75. Moolgavkar S, Luebeck G, Anderson E. Air pollution and hospital admissions for respiratory causes in Minneapolis-St. Paul and Birmingham. Epidemiology 1997;8:364-370.

76. Schwartz, J. 0 zone, PM 10, and hospital admissions for the elderly in Minneapolis-St Paul, Minnesota. Arch Environ Health 1994;49:366-374. 77. Schwartz J, Morris R.Air pollution and hospital admissions for cardiovascular disease in Detroit, Michigan. Am J Epidemiol 1995;142:23-35.

78. Morris R, N aumova E. C arbon monoxide and hospital admissions for congestive heart failure: Evidence of an increased effect at low temperatures. Environ Health Perspect 1998;106:649-653.

79. Atkinson R, Anderson H, Strachan D, Bland J, Bremner S, Ponce de León A. Short term associations between outdoor and visits accident and emergency departments in London for respiratory complains. Eur Respir J 1999;13:257-265.

80. Damakosh A, Castillejos M, Bierzwinski S, Retama A, Gold D. Acute effects of air pollution on emergency room visits for respiratory disease in children living in South W est Mexico City. In Press 2000.

81. Samet J. Particulate air pollution and mortality:The Philadelphia story. Epidemiology 1995; 6:471-473.

82. 0 stro B, Lipsett M, Mann J. Air pollution and asthma exacerbations among african-american children in Los Angeles. Inhal Toxicol 1995;7: 711-722.

83. Roemer W, Hoek G, Brunekreef B. Effect of ambient winter air pollution on respiratory health of children with chronic respiratory symptoms. Am Rev Respir Dis 1993;147:118-124.

84. N eukirch F, Segala C, Moullec Y, Korobaeff M, Aubier M. Short-term effects of low-level winter pollution on respiratory health of asthmatic adults. Arch Environ Health 1998;53:320-328.

85. Schwartz J, Slater D, Larson T, Pierson W. Particulate air pollution and hospital emergency room:Visits for asthma in Seattle.Am Rev Respir D is 1993;147:826-831.

86. Gielen H,Van der Zee S,W ijnen J, Steen C, Brunekreef B.A cute effects of summer air pollution on respiratory health of asthmatic children.Am J Respir C rit Care 1997; 155: 2105-2108.

87. Peters $A, D$ ockery $D, W$ ichmann $H$. Short-term effects of particulate air pollution on respiratory morbidity in asthmatic children. Eur Respir J 1997;10:872-879.

88. Dusseldorp A, Kruize $H$, Brunekreef $H$, Hofschreuder $P$, Meer $G$, Van 0 udvorst A. Associations of PM10 and airborne iron with respiratory health of adults living near a steel factory. Am J Respir Crit Care Med 1995;152:1939-9.

89. Peters A, Dockery D, Heinrich J,W ichmann E. Medication use modifies the health effects of particulate sulfate air pollution in children with asthma. Environ Health Perspect 1997;105; 430-435.

90. Pope III A, D ockery D.A cute health effect of PM 10 pollution on symptomatic and asymptomatic children. Am Rev Respir D is 1992;145: 1123-1128.

91.A bbey D, Petersen F, Mills P, Beeson W. Long-term ambient concentrations of total suspend particulates, ozone, and sulfur dioxide and respiratory symptoms in a nonsmoking population. Arch Environ Health 1993;48(1):33-46.

92. Peters A,W ichmann H, Tuch T, H einrich J, Heyder J. Respiratory effects are associated with the number of ultrafine particles. Am J Respir Crit Care 1997;155:1376-1383.

93. Schwartz J. Particulate air pollution and chronic respiratory disease. Environ Res 1993;62:7-13.

94. Pope III A. Respiratory hospital admissions associated with PM ${ }_{10}$ pollution in Utah, Salt Lake, and Cache Valleys. Arch Environ Health 1991; 46(2):90-97.

95.A unan K. Exposure-response functions for health effects of air pollutants based on epidemiological findings. Risk Analysis 1996;16:693-709.
96. D ockery D, Speizer F, Stram D,W are J, Spenmgler J, Ferris B. Effects of inhalable particles on respiratory health of children. Am Rev Respir D is 1989;139:587-594

97. Chestnut L, Schwartz J, Savitz D, Burchfiel C. Pulmonary function and ambient particulate matter : Epidemiological evidence from N HAN ES.A rch Environ Health 1991;46:135-144.

98. D ockery D,W are J, Ferris B, Speizer F, C ook N, Herman S. C hange in pulmonary function in children associated with air pollution episodes. J Air Poll Control Assoc 1982;32:937-942.

99. Hoek $G$, Brunekreef B.Acute effects of a winter air pollution episode on pulmonary function and respiratory symptoms of children. Arch Environ Health 1993;48:328-335.

100. Koe N ig J, Larson T, Hanley Q, Rebolledo V, D umler K, Checkoway H et al. Pulmonary function changes in children associated with fine particulate matter. Environ Res 1993;63:26-38.

101. Pope III A, Kanner R.Acute effects of PM 10 on pulmonary function of smokers with mild to moderate chronic obstructive pulmonary disease. Am Rev Respir Dis 1993;147:1336-1340.

102. H oek G, Brunekreef B. Effects of low-level winter air pollution concentrations on respiratory health of Dutch children. Environ Res 1994; 48:328-335.

103. Gold D, D amakosh A, Pope III A, D ockery D, MCD onell W, Serrano P et al. Particula and ozone pollutant effects on the respiratory functions of children in Southwest Mexico City. Epidemiology 1999;10:8-16.

104. 0 stro B. Associations between morbidity and alternative measures of particulate matter. Risk Analysis 1990;10(3):421-427.

105. Loomis D, Borja-A burto V, Bangdiwala S, Shy C. 0 zone exposure and dailly mortality in Mexico City: A time-series analysis. Invest Rep 1996; 75:1-45.

106. Borja-A burto V, C astillejos M, Gold D, Bierzwinski S, Loomis D. Mortality and ambient fine particles in Southwest Mexico City, 1993-1995. Environ Health Perspect 1998;106:849-855.

107. Hoek G, Schwartz J, G root B, Eilers P. Effects of ambient particulate matter and ozone on daily mortality in Rotterdam, the $\mathrm{N}$ etherlands.Arch Environ Health 1997;52:455-463.

108. Kinney $\mathrm{P}, \mathrm{O}$ zkaynak $\mathrm{H}$. Associations of daily mortality and air pollution in Los Angeles County. Environ Res 1991;54:99-120.

109. Touloumi G, Katsouyanni K, Zimurou D, Schwartz J, Spix C. Shortterm effects of oxidant: A combined analysis within the APHEA project. Am J Epidemiol 1997;146:177-185.

110. Schwartz J.Air pollution and hospital admissions for respiratory disease. Epidemiology 1996;7:20-28.

111. Schwartz J. Air pollution and hospital admissions for the elderly in Detroit, Michigan. A m J Respir C rit C are 1994;150:648-655.

112. Téllez-Rojo M, Romieu I, Polo-Peña M, Ruiz-Velasco S, MenesesGonzález F, Hernández-A vila M. Efecto de la contaminación ambiental sobre las consultas por infecciones respiratorias en niños de la ciudad de México. Salud Publica Mex 1997:39:513-522.

113. Burnett R, Dales R, Raizenne M, Krewski D, SummersW, Roberts $G$ et al. Effects of low levels of ozone and sulphates on the frequency of respiratory admissions to 0 ntario hospitals. Environ Res 1994;65: 172194.

114. Stieb D, Burnett R, Beveridge R, Brook J.Association between ozone and asthma emergency department visits in Saint John, N ew Brunwick, Canada. Environ Health Perpect 1996;104:1354-1360.

115. Peters J, A vol E, N avidi W, London S, G auderman J, Lurman F et al. A study of twelve Southern $C$ alifornia communities with differing levels and types of air pollution. Am J Respir C rit C are 1999;159:760-767.

116. 0 laiz G, Sánchez I, Rojas M,Torres V, Mendoza L. Evaluation of health and air pollution indexes in Mexico city. In press 2000. 
117.W ilson R, Spengler J. Particles in our air. Cambridge: Harvard U niversity Press, 1996:85-168.

118. International C ommission on Radiological Protection. Task force on lung dynamics: Deposition and retencion models for internal dosimetry of the human respiratory tract. Health Physics 1966;12:173-207.

119. Ghio J, Samet J. Metals and air pollution particles. En: Holgate ST, Saneet SM, Maynard RL, Borel HS, ed.Air pollution and health. San D iego (CA): A cademic Press, 1999:635-651.

120. Brauer M, Dumyahn T, Spengler D, Gutshmidt K, Heinrich J, W ichmann H. Measurement of acidic aerosol species in eastern Europe: Implications for air pollution epidemiology.J 0 ccup Environ Med 1995;103: 482-488.

121. Pope CA, D ockery D w, Kanner RE, Villegas GM, Schwartz J. 0 xygen saturation, pulse rate, and particulate air pollution: A daily time- series panel study.Am J Respir C rit Care Med 1999;159:365-372.

122. Peters A, D oring A, W ichmann HE, Koening W . Increased plasma viscosity during the 1985 air pollution episode: A link to mortality? Lancet 1997;349:1582-1587.
123. Peters A, Persz S, D oring A, Stieber J, Koening W. Increases in heart rate during an air pollution episode. Am J Epidemiol 1999;150:1094-1098. 124. Liao D, C reason J, Shy C, W illiams R, Z weidinger R. D aily variation of particulate air pollution and poor cardiac autonomic control in the elderly. Environ Health Perspect 1999;107(7):521-525.

125. Pope CA, Verrier RL, Lovett EG, Larson AC, Raizenne ME. Kanner RE et al. Heart rate variability associated with particulate air pollution. Am Heart J 1999; 138:890-899.

126. N olan J, Batin PD, A bdrewusR. Prospective study of heart rate variability and mortality in chronic heart failure. Circulation 1998;98:15101516.

127. Bromberg P. Structure-functions relationships in air pollution and health. San Diego (CA):Academic Press, 1999: 269-294. 